\title{
Three-dimensional trajectories of cultivated Pacific bluefin tuna Thunnus orientalis in an aquaculture net cage
}

\author{
Kazuyoshi Komeyama ${ }^{1, *}$, Minoru Kadota ${ }^{2,3, *}$, Shinsuke Torisawa ${ }^{2}$, \\ Tsutomu Takagi ${ }^{2, * *}$
}

\author{
${ }^{1}$ Faculty of Fishery, Kagoshima University, 4-50-20 Shimoarata, Kagoshima 890-0056, Japan \\ ${ }^{2}$ Department of Fisheries, Faculty of Agriculture, Kinki University, 3327-204 Naka-machi, Nara 631-8505, Japan \\ ${ }^{3}$ Temple University, 4-1-27 Mita, Minato-ku, Tokyo 109-0073, Japan
}

\begin{abstract}
Swimming trajectories of aquatic animals that are estimated using the deadreckoning technique below the sea surface tend to have very large associated observational errors. Therefore, the aim of the present study was to develop a technique for removing accumulated errors from such trajectories for Pacific bluefin tuna Thunnus orientalis. Horizontal and vertical speeds and heading angle were measured in an aquaculture net cage using 2 types of data loggers, and current velocity was recorded at a depth of $12 \mathrm{~m}$ to measure the tidal current speed around the net cage. Fourier analysis indicated that the primary source of error in trajectory estimates was the effect of ocean currents, which resulted in drift, and further analysis revealed that the frequency contributing to drift was consistent with the low-frequency signal in a spectrum analysis of horizontal speed. Therefore, a high-pass filter was applied to horizontal speed data to remove any frequencies lower than the cut-off frequency $(0.0015 \mathrm{~Hz})$, following which these data were back-transformed into a time domain that no longer included the drift effect caused by the current. The reconstructed trajectories fit within the inner diameters of the net cage, indicating that they were realistic. To confirm the validity of the resultant swimming trajectories, a flume tank experiment was conducted, which demonstrated that the high-pass filter effectively removed current drift from the estimated trajectory. Furthermore, since the method was estimated to have a precision of approximately $0.20 \mathrm{~m}$, it not only allows the 3-dimensional trajectories of circling tuna to be estimated but can also be applied to the behavior of fish in the wild.
\end{abstract}

KEY WORDS: Dead reckoning $\cdot$ Behaviour $\cdot$ Submerged net cage

\section{INTRODUCTION}

Aquaculture net cages for Pacific bluefin tuna Thunnus orientalis are currently designed to withstand rough conditions from ocean currents and waves (Suzuki et al. 2009) but do not meet the biological requirements of this species. Because cultivated tuna swim faster than other fishes, the shape, volume, and dimensions of these cages need to be adequate to allow tuna to swim and turn, as it is likely that the need to make sharp turns would stimulate

*These authors contributed equally to this work

${ }^{* *}$ Corresponding author. Email: tutakagi@nara.kindai.ac.jp stress, reducing the quality of cultivated fish. Therefore, scientific information is required to determine the most suitable cage radius to allow circling fish to turn along cage walls and to determine how tuna swim in all 3 dimensions within the cages, particularly since net cages are altered into various 3dimensional (3D) shapes by the tidal current (Suzuki et al. 2009). Visualization of the 3D behavior of fish provides data on their turning angle, the radius of the swimming circle, and the space used by individuals within the cage. However, to complete such an

(C) The authors 2013. Open Access under Creative Commons by Attribution Licence. Use, distribution and reproduction are unrestricted. Authors and original publication must be credited.

Publisher: Inter-Research · www.int-res.com 
analysis, new methods are needed to allow accurate measurement of the 3D trajectories of bluefin tuna.

A complete understanding of fish behavior below the sea surface remains elusive due to limitations of the available technology for making indirect underwater observations in both natural and enclosed areas. Although some studies have described the behaviors of cultivated tuna in aquaculture net cages (Kubo et al. 2004, Okano et al. 2006, Kadota et al. 2011), these have relied on measurements of $1 \mathrm{D}$ swimming behavior, swimming depth, acceleration, and/or feeding behavior.

Two techniques are currently available for examining the movements of aquatic animals below the sea surface: acoustic telemetry (e.g. Hindell et al. 2002) and dead reckoning (Wilson \& Wilson 1988, Shiomi et al. 2008, Komeyama et al. 2011). Acoustic telemetry estimates the position of fish using acoustic transmitters that measure the difference in time the emitted sound takes to arrive at hydrophones (e.g. Harcourt et al. 2000, Hindell et al. 2002, Bégout Anras \& Lagardère 2004). This method has several drawbacks, including that a receiver must be located within a few hundred meters of the target fish, making it difficult to obtain data for species with extensive ranges (Wilson et al. 2007); it is difficult to affix acoustic receivers in offshore areas; ocean waves and oscillations of the hydrophones by waves generate acoustic noise; changes in water temperature in the thermocline strongly affect the acoustic velocity; also, it is difficult to continuously detect the signal at a fine scale (i.e. a few seconds) to estimate high-resolution fish trajectories. Thus, although acoustic telemetry has the advantage of directly measuring the position of fish, it may be difficult to measure fish behavior with a high accuracy and over a fine time scale in offshore areas.

The dead-reckoning technique overcomes these problems by using a very simple principle. Specifically, it requires data on a target's speed, heading angle, and depth change per given interval; velocity vectors are then calculated and estimated indirectly based on an observational dataset, following which 3D trajectories can be constructed. Thus, dead reckoning produces temporally resolved, regular, sequential positional data with no gaps (Wilson et al. 2002). However, this method also has drawbacks in that the trajectories include cumulative error from multiple sources. Some of this error is associated with the lack of underwater reference points, but the greatest source of inaccuracy originates from drift resulting from factors such as ocean currents and winds (Bramanti et al. 1988, Mitani et al. 2003, Shiomi et al. 2008, Komeyama et al. 2011), and since a trajectory is estimated by integrating velocity vectors, this inaccuracy increases with time.

Komeyama et al. (2011) measured the 3D trajectories of bluefin tuna in a submerged aquaculture net cage and used linear detrending to remove accumulated error from the reconstructed trajectories. However, the details of the trajectories were inadequate, and so only limited information could be estimated because almost all of the reconstructed trajectories drifted nonlinearly. In addition, there were no clear grounds for detrending the linear component from the nonlinear drift, leaving room for improvement.

In the present study, we assessed the potential sources of drift error due to ocean currents and developed a new technique for removing this error to allow for a more accurate visualization of the trajectories of circling cultivated bluefin tuna in an aquaculture net cage. To do this, we reanalyzed the behavioral data described by Komeyama et al. (2011), to allow direct comparison with their results. We attempted to reconstruct the 3D trajectories of tuna, which were calculated using a high-pass filter method, and then confirmed the validity of the method by conducting an experiment in a large flume tank.

\section{MATERIALS AND METHODS}

In the present study, we carefully analyzed the time series of ocean current data that were collected in an experimental net cage by Komeyama et al. (2011), using a high-pass filter to remove the drift signal (which decreases slowly). To test the validity of using this high-pass filter method within the deadreckoning technique, we confirmed the calculated trajectories using both a field and laboratory experiment.

\section{Formulation of the dead-reckoning technique}

The dead-reckoning technique was used to visualize the swimming paths of a single tuna in an aquaculture net cage. Velocity vectors were estimated based on speed $(v)$, heading angle $(\theta)$, and change in depth $(d)$ per measurement interval $(t)$ using the following formulae:

$$
\begin{gathered}
v_{x_{t}}=\sqrt{v_{t}^{2}-v_{z_{t}}^{2}} \cdot \cos \theta_{t} \\
v_{x_{t}}=\sqrt{v_{t}^{2}-v_{z_{t}}^{2}} \cdot \sin \theta_{t} \\
V_{z_{t}}=d_{t}-d_{t-1}
\end{gathered}
$$


where $\Delta t=1$ corresponds to the measurement inter$\mathrm{val}$, and the vector $\left(V_{x_{t}}, V_{y^{\prime}}, V_{z_{t}}\right)$ is a locomotion vector at measurement time $t$. The 3D swimming path could then be reconstructed from the observational dataset by integrating the locomotion vector with respect to time (i.e. summing Eqs. 1 to 3 ) to give the following:

$$
\begin{gathered}
x_{t}=\sum_{n=1}^{N} \sqrt{v_{t}^{2}-v_{z_{t}}^{2}} \cdot \cos \theta_{t} \\
y_{t}=\sum_{n=1}^{N} \sqrt{v_{t}^{2}-v_{z_{t}}^{2}} \cdot \sin \theta_{t} \\
z_{t}=\sum_{n=1}^{N}\left(d_{t}-d_{t-1}\right)
\end{gathered}
$$

However, the horizontal swimming paths obtained using Eqs. (4) and (5) include large cumulative errors (Shiomi et al. 2008, Komeyama et al. 2011), mostly due to the horizontal ocean current. By contrast, the vertical swimming path given by Eq. (6) includes less cumulative error because the magnitude of vertical currents is much lower than that of the horizontal current. Therefore, Eqs. (4) and (5) were decomposed into 3 terms (the tuna-location term, the current-drift term ( $c d$ ), and the accumulation of error term) and then solved for tuna location. Thus, the tuna location at measurement time $t$ can be written as follows:

$$
\begin{gathered}
x_{\text {tuna }, t}=\sum_{n=1}^{N} \sqrt{v_{t}^{2}-v_{z_{t}}^{2}} \cdot \cos \theta_{t}+x_{c d, t} X_{\varepsilon, t} \\
y_{\text {tuna }, t}=\sum_{n=1}^{N} \sqrt{v_{t}^{2}-v_{z_{t}}^{2}} \cdot \sin \theta_{t}+y_{c d, t}+y_{\varepsilon, t}
\end{gathered}
$$

where $x_{\varepsilon, t}$ and $y_{\varepsilon, t}$ represent observational errors, and $x_{u}$ and $y_{u}$ represent errors associated with horizontal current velocities $(u)$. Thus, the net errors can be expressed as follows:

$$
\begin{aligned}
& x_{c d, t}=\sum_{n=1}^{N} x_{u} \\
& y_{c d, t}=\sum_{n=1}^{N} y_{u}
\end{aligned}
$$

To correct the estimated positional data, the net errors of Eqs. (9) and (10) were then removed by assuming constant linear drift. However, positions estimated in this way accumulate error with time and become more inaccurate simply because the ocean current is not at all constant. Therefore, we required alternative techniques that do not assume constant current velocities to remove errors associated with ocean currents.

\section{Removing low-frequency drift}

The summed observational data calculated using Eqs. (4) \& (5) include low-frequency drift, likely as a result of physical noise, the major cause of which is the ocean current (corresponding to the second terms in Eqs. 7 \& 8). If these biases due to physical noise are not accounted for, they invalidate events related to the biological signals of interest and substantially decrease the power of the statistical analysis. Therefore, the removal of low-frequency drift is one of the most important steps in reconstructing 3D fish trajectories. Unfortunately, this pre-processing step is also one of the most dangerous steps because the biological signal of interest may easily be removed if incorrect filters are applied. To test the developed equations and data treatment, in situ measurements were required.

\section{Reconstructing swimming trajectories in a net cage}

We conducted an experiment in a submerged net cage installed in offshore waters of Kochi Prefecture, Japan, to reanalyze the data of Komeyama et al. (2011). The diameter of the cage was $30 \mathrm{~m}$, and the net was completely submerged, with the ceiling located at a depth of $2 \mathrm{~m}$ and the floor at $\sim 22 \mathrm{~m}$ below the surface.

A single bluefin tuna (fork length: $51 \mathrm{~cm}$; estimated weight: $2.6 \mathrm{~kg}$ taken from a regression line fit to tuna-farm records) was captured from within the cage by angling. Two micro-data loggers (PD3GT, Little Leonardo, $75 \mathrm{~g}$ in air; DST Comp-Tilt, StarOddi, $19 \mathrm{~g}$ in air) that had been inserted into a single floating cellular material plate $(9 \mathrm{~cm}$ long and $3.5 \mathrm{~cm}$ high), the buoyancy of which had been adjusted to slightly more than its underwater weight, were externally attached to the body of the tuna near the second dorsal fin (Komeyamaet al. 2011). The fish was then released back into the net cage.

The PD3GT data logger recorded swimming speed and depth at $1 \mathrm{~s}$ intervals on a flash memory drive from 09:30 h on 6 March to 17:30 h on 7 March 2010. A propeller was attached to the PD3GT to record the speed through the water, and we confirmed the relationship between velocity and the number of propeller revolutions as well as the stall speed of the device ( 0.13 to $0.18 \mathrm{~m} \mathrm{~s}^{-1}$ ) in a preliminary experiment. In the study itself, the tagged fish swam at speeds of $>0.28 \mathrm{~m} \mathrm{~s}^{-1}$ without the propeller stopping.

The DST Comp-Tilt data logger recorded the heading of the fish, which was calculated using 2D geo- 
magnetism at $1 \mathrm{~s}$ intervals. Due to the logger's limited memory capacity, data from the DST Comp-Tilt were divided into 4 phases of daily activity: dawn, 05:0007:00 h; daytime, 11:30-12:30 h; dusk, 17:00-19:00 h; and night-time, 23:30-24:30 h. A summary of the depths and turning angles of the tagged fish during each phase is presented in Table 1. Initially, we hypothesized that rotation of the propeller on the PD3GT data logger would influence these measurements, as the rotation speed of the propeller was transmitted through magnetic variation caused by a magnet attached to the propeller shaft. However, our preliminary experiment detected no significant influence of this on compass measurements. The data loggers were detached from the fish using a timing device and were collected by a diver after $3 \mathrm{~d}$.

To monitor the current profile in the net cage, an electromagnetic current meter (Infinity-EM; JFE Advantec) was affixed to the outside of the net cage at a depth of $12 \mathrm{~m}$ from $14: 30 \mathrm{~h}$ on 5 March until 10:00 h on 12 March 2010. The meter recorded the current speed and direction, which were calculated from the $2 \mathrm{D}$ velocities at $5 \mathrm{~min}$ intervals. To estimate current velocities, 30 samples were taken at $1 \mathrm{~s}$ intervals every $5 \mathrm{~min}$.

To validate the swimming trajectories calculated in the present study, we examined whether the trajectories fit into the aquaculture net cage. We chose the

Table 1. Number of sample, depth, and turning angle of a tagged tuna during each phase. Q1, Q2, and Q3 mean 25, 50, and 75\% quartiles, respectively

\begin{tabular}{|lccccccr|}
\hline $\begin{array}{l}\text { Phase } \\
\text { (h of day) }\end{array}$ & $\mathrm{n}$ & \multicolumn{3}{c}{ Depth (m) } & \multicolumn{3}{c|}{$\begin{array}{c}\text { Turning angle } \\
\text { (degree s }\end{array}$} \\
& & Q1 & Q2 & Q3 & Q1 & Q2 & Q3 \\
\hline $11: 30-12: 30$ & 3600 & 16.36 & 18.99 & 20.55 & 6 & 13 & 22 \\
$17: 00-19: 00$ & 7200 & 17.82 & 19.04 & 19.97 & 6 & 12 & 22 \\
23:30-00:30 & 3600 & 13.91 & 15.43 & 16.65 & 5 & 12 & 23 \\
05:00-07:00 & 7200 & 12.60 & 14.35 & 16.60 & 7 & 16 & 35 \\
\hline
\end{tabular}

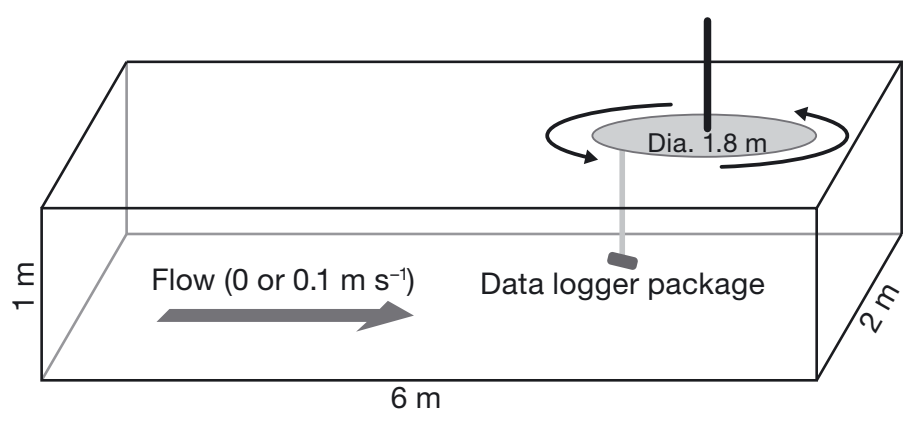

Fig. 1. Schematic illustration of the flume tank experiment origin $(0,0$, depth) as the starting point of a trajectory. Since the net cage had a bowl-like shape, with a maximum diameter of $30 \mathrm{~m}$ near the frame on top of the cage but a gradually decreasing diameter with increasing depth due to the nets being pulled by the sinker, it was difficult to obtain data on the diameter of the cage at each depth. Therefore, we estimated this factor using Net geometry and Loading Analysis (NaLA) software (Takagi et al. 2002, Suzuki et al. 2009), which numerically estimated the geometry and internal forces acting on the net and rigging.

\section{Reconstructing trajectories in a flume tank}

To test the validity of our technique, we also conducted experiments in a flume tank filled with freshwater (Fig. 1). The channel dimensions of the observed area of the flume tank were $6.0 \mathrm{~m}$ length $\times$ $2.0 \mathrm{~m}$ width $\times 1.0 \mathrm{~m}$ depth, and the speeds of the carriages were 0 and $0.1 \mathrm{~m} \mathrm{~s}^{-1}$. We set the PD3GT and DST Comp-Tilt data loggers at the tip of the rod that lay vertically below the edge of a vinyl chloride disk (diameter: $1.8 \mathrm{~m}$ ), which completed a lap over $\sim 20 \mathrm{~s}$ at $0 \mathrm{~m} \mathrm{~s}^{-1}$ or $0.1 \mathrm{~m} \mathrm{~s}^{-1}$. After downloading measurement data from these data loggers, both trajectories were reconstructed.

\section{RESULTS AND DISCUSSION}

\section{Reconstructing swimming trajectories in a net cage}

Fourier analysis of the locomotion vector

We conducted a Fourier analysis of the locomotion vector denoted in Eqs. (7) \& (8) to determine the strengths of the respective frequencies of the swimming motion in the data. The power spectra of the $x$-directional (horizontal) component of the locomotion vector $x_{t}$ was obtained by integrating the velocities observed over $1 \mathrm{~h}$ between 23:30 and 00:30 h (Fig. 2a). Two significant peaks appeared in the power spectrum, with the higher-frequency peak $(0.0134 \mathrm{~Hz})$ corresponding to a periodicity of $74 \mathrm{~s}$, which equaled the time needed for a tuna to swim 1 circumference of the net cage. The second frequency peak $(0.0008 \mathrm{~Hz})$ was thought to be the dominant frequency contributing to drift, but it was not clear which frequency should be used as a cut-off to remove the drift error from the 

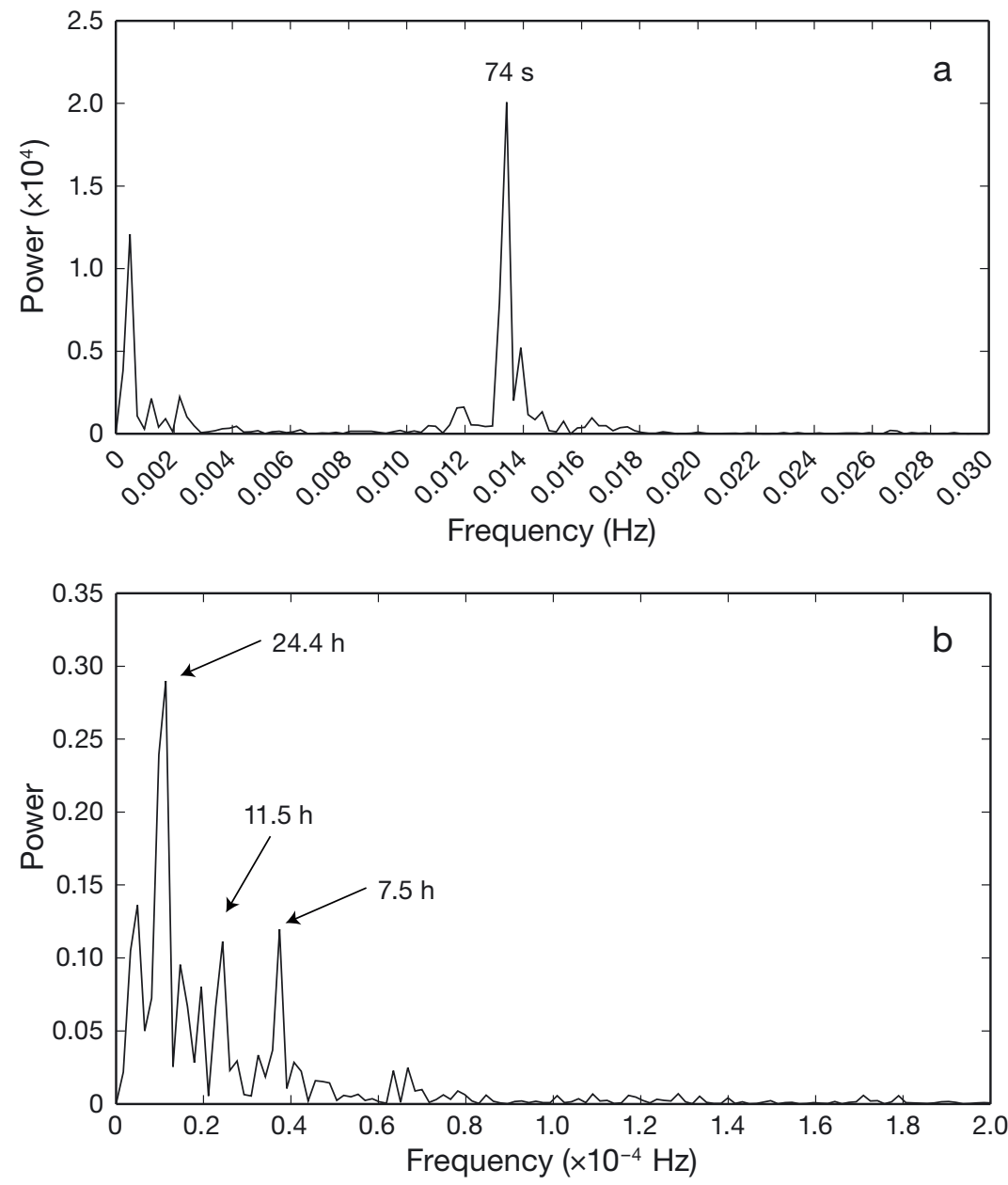

Fig. 2. (a) The power spectra of the $x$-directional component of the locomotion vector $x_{t}$, which was obtained by integrating the observed velocities over $1 \mathrm{~h}$ between 23:30 and 00:30 h. Two significant peaks appear in the frequency spectrum; the higher-frequency peak $(0.0134 \mathrm{~Hz})$ corresponds to a periodicity of $74 \mathrm{~s}$, while the other frequency peak $(0.0008 \mathrm{~Hz})$ is thought to be the dominant frequency contributing to drift. (b) The power spectra of the ocean current at a depth of $12 \mathrm{~m}$ within the net cage. Significant peaks were observed at around $24.4 \mathrm{~h}$ and $11.5 \mathrm{~h}$, with smaller significant peaks at $7.5 \mathrm{~h}$
$11.5 \mathrm{~h}$ and smaller significant peaks at $7.5 \mathrm{~h}$. These spectral peaks were consistent with results reported by Stockwell et al. (2004), who used data from >19000 monthly time series taken from 262 data buoy sites and also discovered peaks at 24,12 , and $8 \mathrm{~h}$ along the frequency spectrum. Thus, our analysis suggested that most of the ocean current power spectrum at a depth of $12 \mathrm{~m}$ was associated with the tidal signal, allowing us to remove the frequency that contributed a major portion of the drift by choosing cut-off values of $<8 \mathrm{~h}$. It should also be noted that ocean currents fluctuate over short periods, but this high-frequency fluctuation was ignored as noise for the purpose of reconstructing the $3 \mathrm{D}$ tuna trajectories in the present study, as we were only concerned with a time scale of a few minutes to a few hours. Thus, we calculated the energy that was concentrated at a frequency greater than $0.00021 \mathrm{~Hz}$ (the fourth peak in Fig. 2a) and found that $31 \%$ of the ocean current energy occurred at these higher frequencies. This fluctuation in ocean current energy needed to be removed from our observational data; therefore, we reanalyzed the data used by Komeyama et al. (2011) from a frequency perspective and considered the role of high frequencies to select an appropriate cut-off frequency. data. Therefore, we conducted a more thorough analysis of the ocean current.

Fourier analysis of the ocean current

We conducted a Fourier analysis on the time series of ocean current data to estimate which frequency contributed to drift. The power spectra of the ocean current at a depth of $12 \mathrm{~m}$ within the net cage were calculated over $6 \mathrm{~d}$, including the day on which the experiment was conducted (Fig. 2b). Although the dataset was not large, our analysis resolved the tidal signatures, with significant peaks at $\sim 24.4 \mathrm{~h}$ and

\section{Reconstructed 3D trajectories and their validity}

When reconstructing the 3D swimming paths of bluefin tuna, Komeyama et al. (2011) assumed a constant linear drift over time and thus subtracted drift error by means of the least-squares fit. However, external effects such as ocean currents vary over time, invalidating the linear drift assumption and leading to large errors in the calculated swimming paths. Therefore, we compared the linear drift and high-pass filter methods and evaluated the nonlinear components of the drift error from a frequency perspective.

Given the highly linear relationship between the $x$-component of the locomotion vector and time 
(Fig. 3a), Komeyama et al. (2011) fit a linear trend to the data and removed the accumulated error to give a corrected swimming path (red line in Fig. 3b). The blue line is the $x$-component of the locomotion vector that was removed by the high-pass filter with a cutoff frequency of 0.00021 (Fig. 3b). Neither of these methods gave trajectories that fit within the diameter of the cage (maximum $30 \mathrm{~m}$ ), as the maximum and minimum values for both methods were greater than $15 \mathrm{~m}$ and less than $-15 \mathrm{~m}$, respectively. This suggested that a nonlinear effect due to a higher frequency range $(>0.00021 \mathrm{~Hz})$ needed to be removed to reduce the amplitude of the trajectories. Therefore, we estimated the appropriate cut-off values based on the above analysis and used a high-pass filter to remove all frequencies contributing to drift in the observational data.

We conducted a Fourier analysis to estimate which frequencies potentially contributed to drift. All frequencies less than $0.0015 \mathrm{~Hz}$ were removed by a
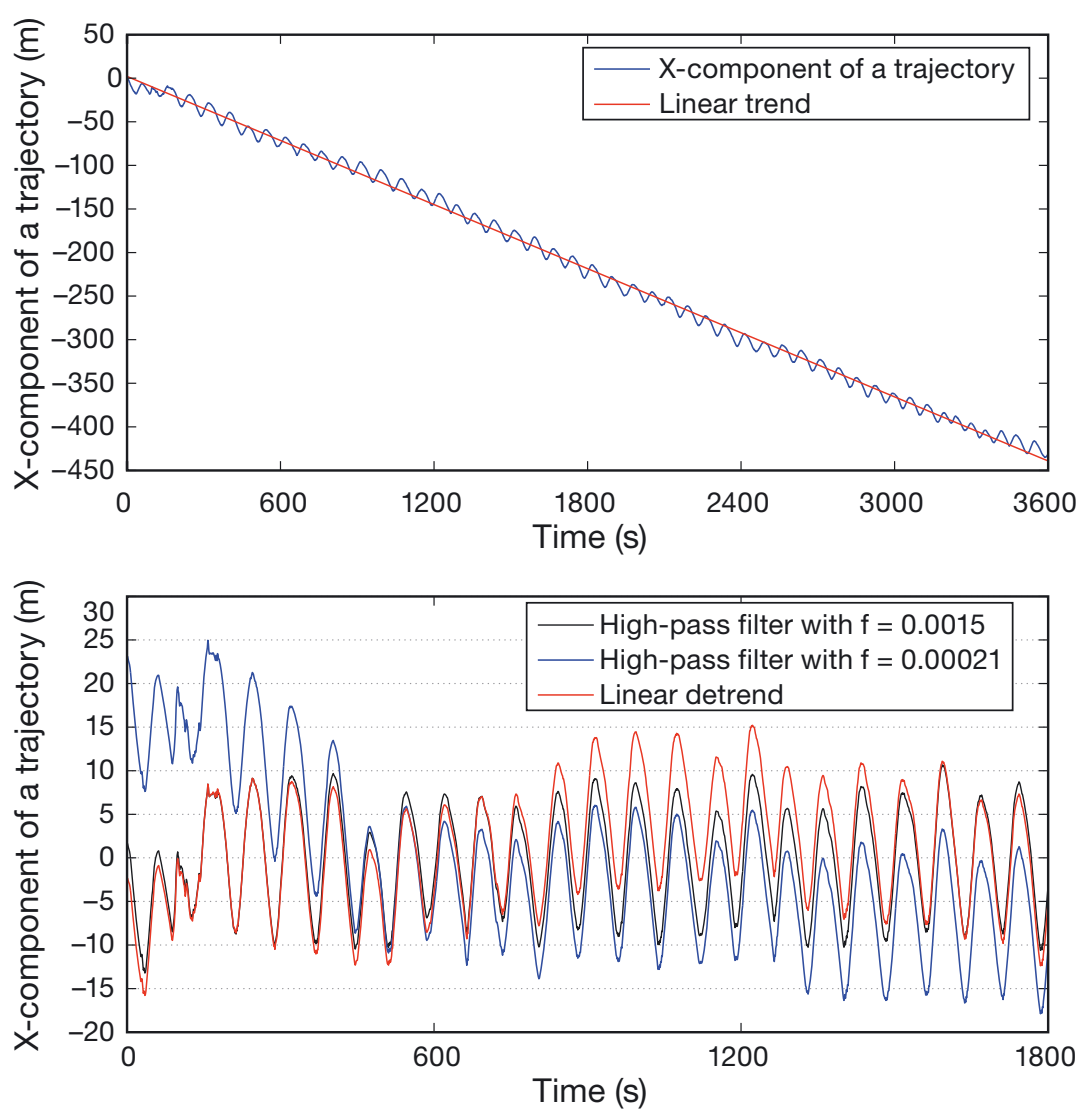

Fig. 3. (a) The liner trend in the $x$-component of the locomotion vector through time. (b) Corrected trajectories were calculated by subtracting a linear trend or using the designed high-pass filter. The maximum and minimum values are denoted by the red line (linear square regression method) and blue line (the high-pass filter with a cut-off frequency of $0.00021 \mathrm{~Hz}$ ), respectively. The black line is the low-frequency $(<0.0015 \mathrm{~Hz})$ signal in the $x$-component of the locomotion vector that was removed by the high-pass filter high-pass filter designed in the MATLAB Signal Processing Toolbox. The resultant data were then backtransformed into the time domain. The low-frequency $(<0.0015 \mathrm{~Hz})$ part of the $x$-component of the locomotion vector was removed by the high-pass filter, and the filter successfully removed drift from the data (Fig. 3b). Therefore, we applied the same filter to all of the observational data, removing any drift due to low-frequency movement. The amplitude of the $x$-component of the 3D trajectory derived using this method did not exceed the radius of the net cage (Fig. 3).

We reconstructed trajectories for night-time data (Fig. 4). We chose the origin as the starting point of each trajectory, but any point could have been selected without affecting the shape of the trajectory, swimming speed, or heading of the fish (although the distance between the fish and the cage wall may have changed). The calculated trajectories fit within the maximum diameter $(30 \mathrm{~m})$ of the net cage. We then calculated the trajectories for other time periods (dawn, daytime, and dusk) in the same manner as the reconstructed trajectories and found that they also fit within the maximum diameter of the net cage (Fig. 5a). For example, trajectories that represented the tuna swimming between the middle and the bottom of the cage during the daytime (Table 1) fit within the diameter of the net cage at each depth (Fig. 5b). Although partial trajectories that extended beyond the cage were observed, these could be explained by observational error in Eqs. (7) \& (8). Thus, the modified trajectories represent more realistic estimates than those presented by Komeyama et al. (2011) (Figs. 4 \& 5).

\section{Reconstructing trajectories in a flume tank}

To validate the high-pass filter method, we conducted a Fourier analysis to estimate which frequencies potentially contributed to drift and, because the disk was rotating at $\sim 0.04$ to $0.05 \mathrm{~Hz}$, removed all frequencies less than $0.03 \mathrm{~Hz}$ using the high-pass filter method described for the field experiment. The data were then 

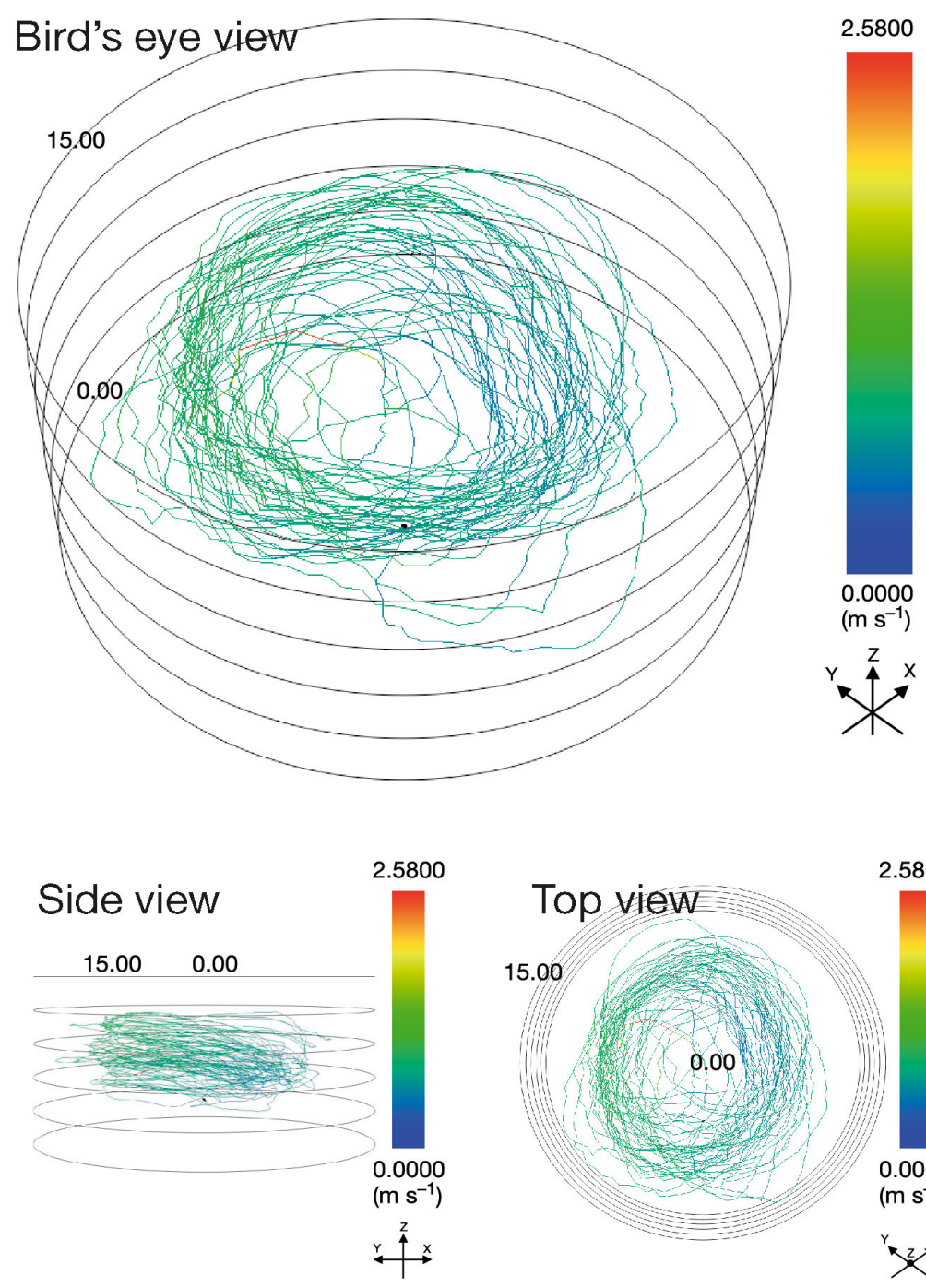

Fig. 4. Night-time (23:30 to $0: 30 \mathrm{~h})$ trajectories as reconstructed by the methods proposed in the present study. Colors of the trajectories indicate swimming speeds

back-transformed into the time domain. We removed the steady flow along with the long-term trend using a high-pass filter (Fig. 6). The filtered trajectories were not perfect circles but did approximate the shape of a circle. Therefore, we calculated the curvature radius of the estimated trajectories using 3 points along each trajectory, which gave radii of 0.80 $\pm 0.17 \mathrm{~m}$ and $0.87 \pm 0.16 \mathrm{~m}($ mean $\pm \mathrm{SD})$ for the $0 \mathrm{~m} \mathrm{~s}^{-1}$ and $0.1 \mathrm{~m} \mathrm{~s}^{-1}$ conditions, respectively. The resulting SDs of 0.16 and $0.17 \mathrm{~m}$ suggested that the precision of this method was $\sim 0.20 \mathrm{~m}$. The curvature radii of the 2 trajectories were similar to but lower than 0.90 $\mathrm{m}$, which is the radius of both true trajectories. This underestimate may have been due to the response of the $x^{-}$and $y$-components having been slightly decreased during filtering. However, Hiraishi (2006) also reported that the propeller rotation sensitivity of

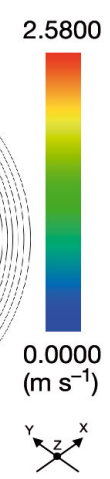

this device decreases drastically with increasing angle of the incoming water, which approaches a tilt of $\sim 20^{\circ}$. In this flume tank experiment, the inclination angle of the propeller in relation to the water flow direction changed by $16^{\circ} \mathrm{s}^{-1}$ when the lap time was $20 \mathrm{~s}$; thus, the propeller rotation may have been less sensitive to a change in the direction of movement due to water flowing over it at this inclined angle. This implies that if a fish turns at a low curvature radius, the dead-reckoning technique using a propeller with attached data loggers may underestimate the diameter of the trajectory. Turning angles greater than the $75 \%$ quartile had less of an effect on trajectories in the flume tank experiment than in the field experiment (Table 1). However, although turning angles of $>20^{\circ}$ occurred a few times in the field experiment, especially during the period 05:00 to 07:00 $\mathrm{h}$, the trajectories calculated in this experiment most likely represented the correct path. Thus, even though the present method may generate some underestimations because the shapes of the filtered trajectories were broken rather than circular (Fig. 6), this is undoubtedly the best currently available method for estimating 3D trajectories.

\section{CONCLUSIONS}

Swimming speed measurements taken by the PD3GT data logger represent speed through the water rather than relative to the ground, making them subject to tidal effects, and the drift trajectory results shown in Fig. 3 imply that the dead-reckoning technique cannot accurately estimate swimming trajectories where there are ocean/tidal currents. Shiomi et al. (2008) applied a dead-reckoning technique similar to the PD3GT used in the present study to reconstruct the 3D trajectories of emperor penguins Aptenodytes forsteri and suggested that the accumulated error was the result of ocean currents, and Mitani et al. (2003) also recognized the influence of current flow when calculating trajectories. However, although these studies provided clear start and end 

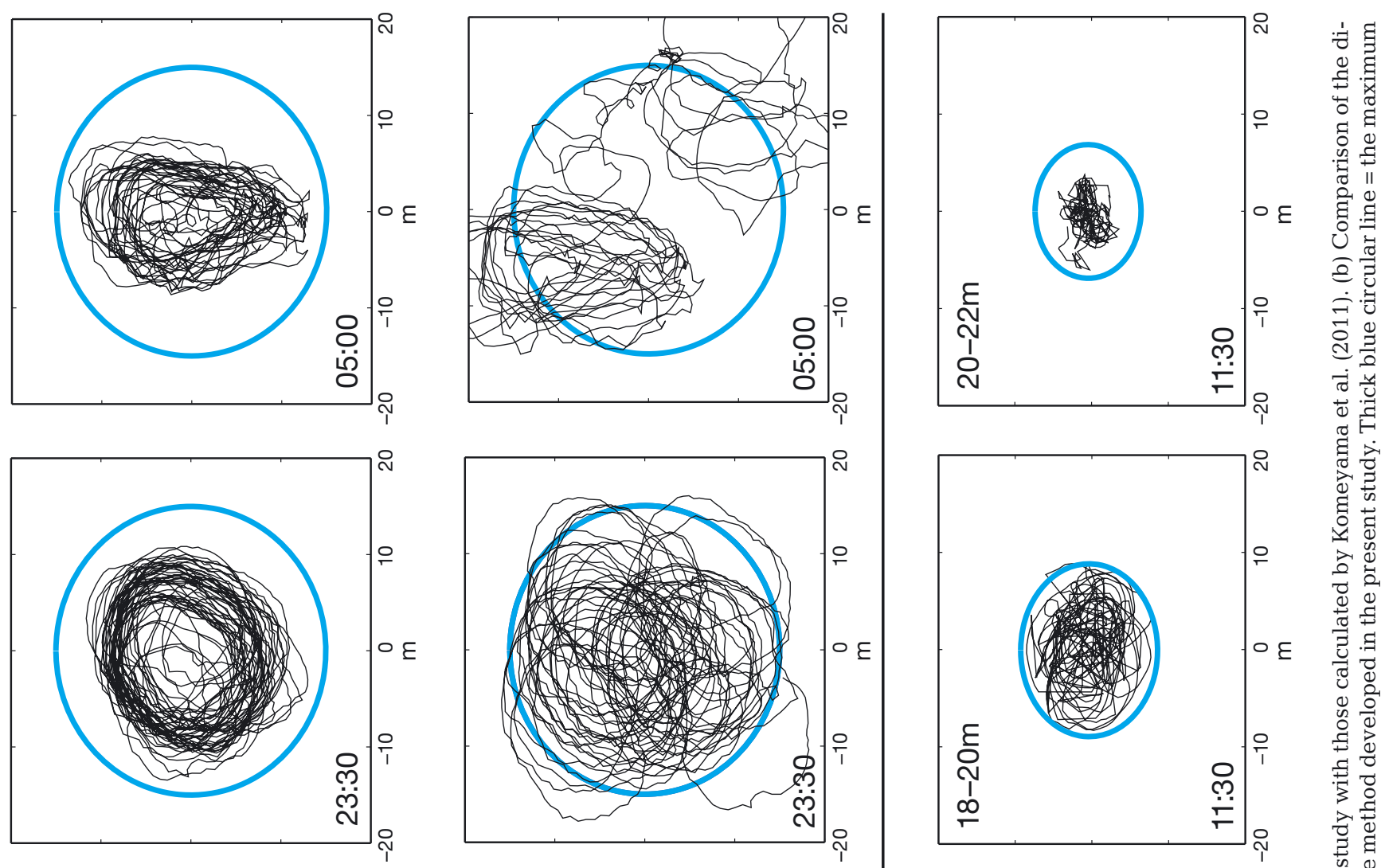

ฮี

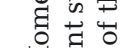
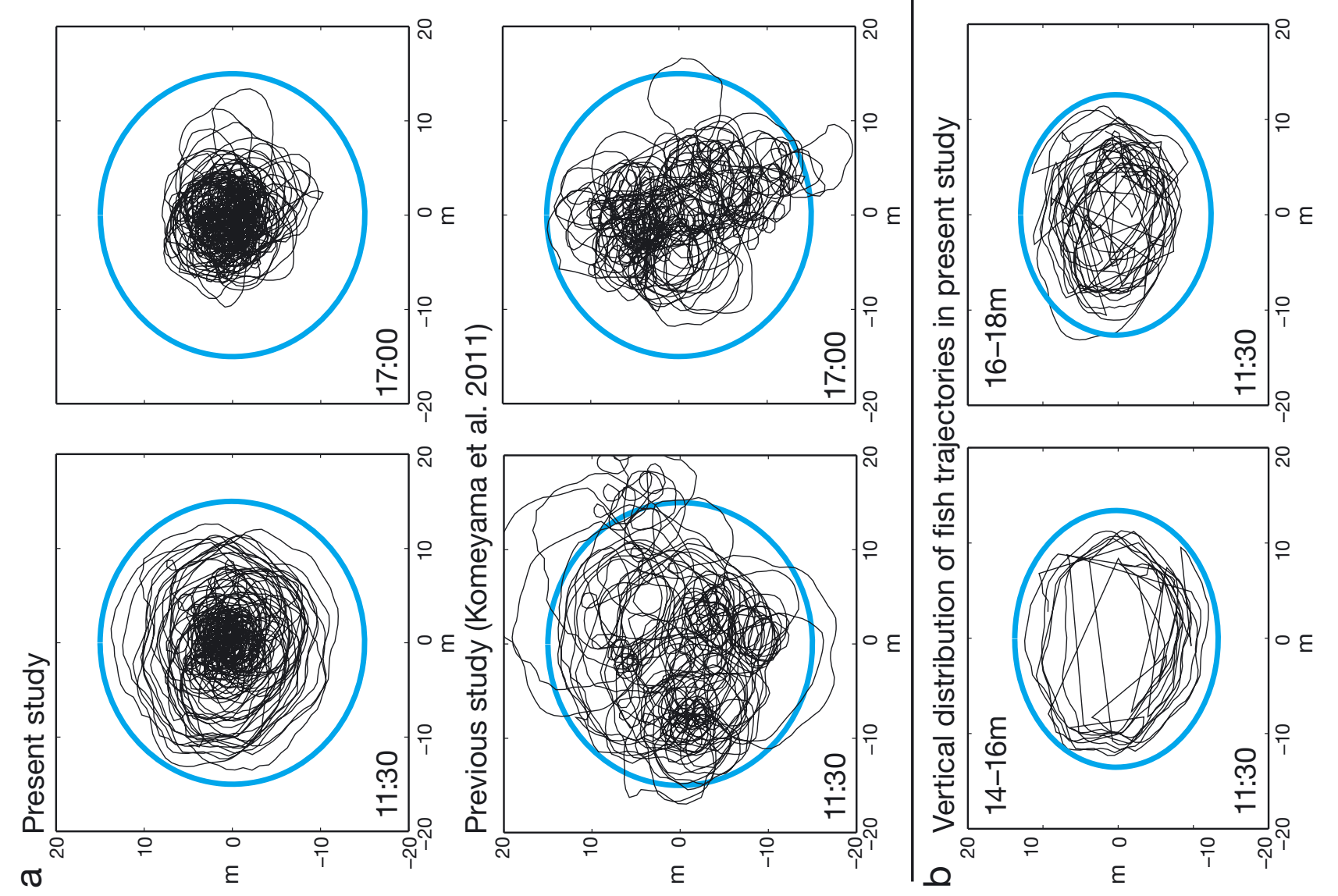

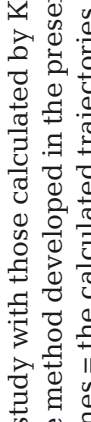

㝋

氖

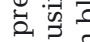

웛

శ్త

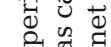

㐘

$\exists$ 要

ป⿱一兀)

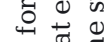

告

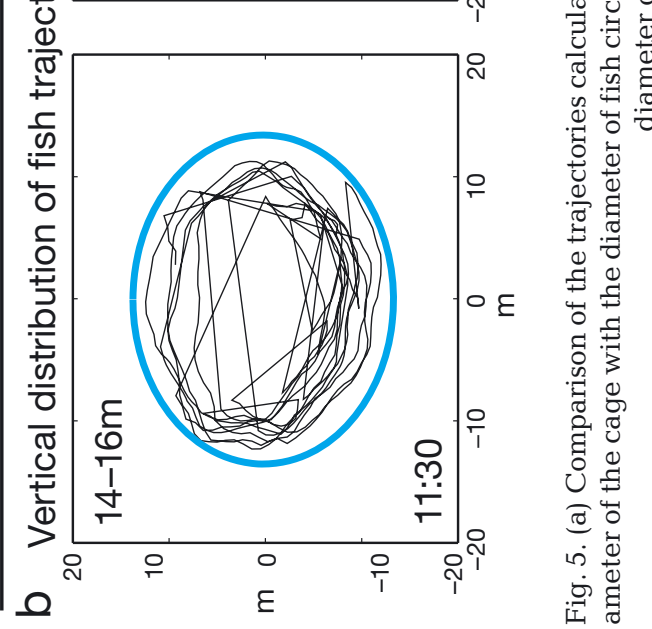



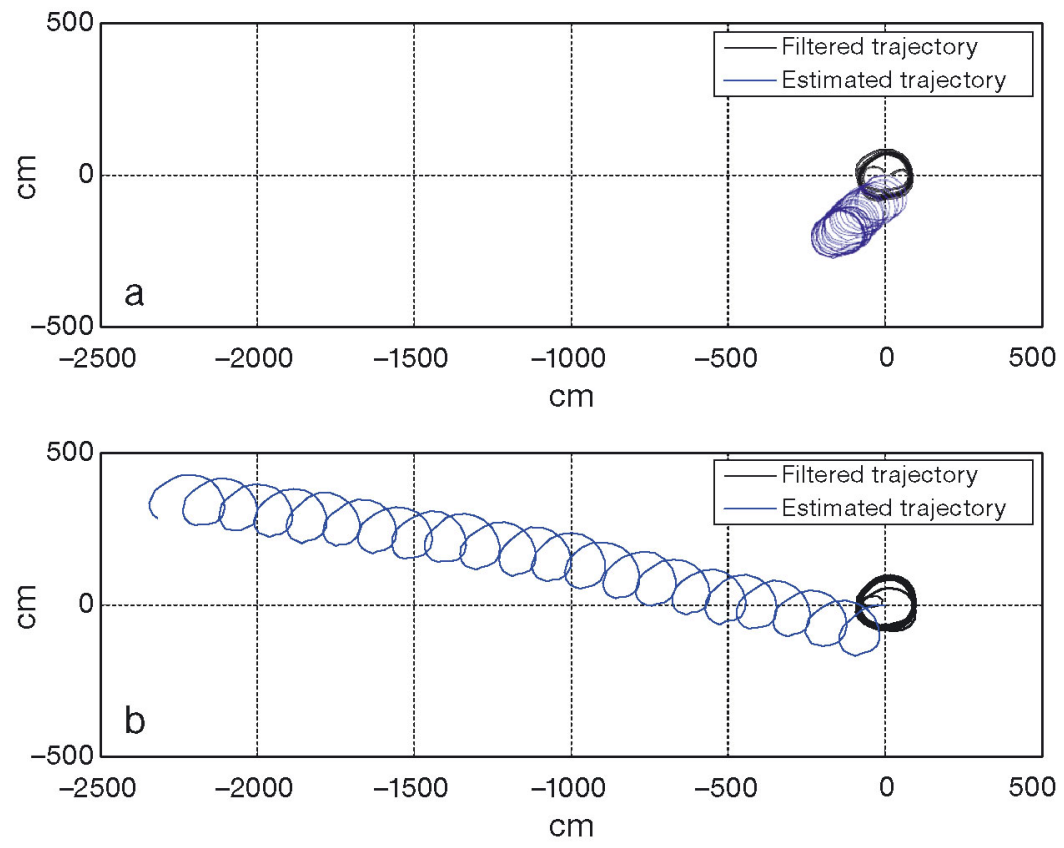

Fig. 6. Results of the flume tank experiment. (a) Water stop, (b) steady flow $\left(10 \mathrm{~cm} \mathrm{~s}^{-1}\right)$. Black lines = reconstructed trajectories; blue lines = corrected trajectories from which accumulated error had been removed using the high-pass filter method

points for the trajectories of animals, the reconstructed end points were not consistent with the actual end points.

In the present study, the fish was in the net cage throughout the observational period. However, in the previous study of Komeyama et al. (2011), the fish appeared to move outside the net cage, despite calm current conditions, making it difficult to determine the exact path taken by the fish because the location would have been influenced by tidal currents. Previous studies have attempted to remove such effects by assuming a linear accumulation of errors; however, although this corrected a portion of the trajectory error, it did not completely remove temporal changes in the tidal component. Moreover, the current can change in 3 dimensions, making it difficult to measure current velocities. By contrast, the high-pass filter method used in the present study was effective at removing accumulated error even when there were $3 \mathrm{D}$ changes in tidal flow, and the results of the flume tank experiment further indicated the usefulness of this method for removing the tidal component and accumulated error. However, the response of the filter was extremely low near the start and end points due to the filter's characteristics. Further research is required to determine how to best overcome this problem (e.g. by excluding the first and last portions of the trajectory from the analysis). However, the methods presented here generated sufficiently accurate trajectories to analyze the turning performance and space use of the net cage by cultivated fish.

By analyzing the frequency of the circling fish in combination with timeseries data for current velocity, we determined the 3D trajectories of a bluefin tuna circling within a submerged aquaculture net cage more accurately than has been accomplished in previous studies. The corrected paths help to determine the swimming speed, inclination of the circle, and swimming depth at which fish swim (Fig. 4), providing useful information regarding the use of space by cultivated fish in aquaculture net cages. Given that no monitoring techniques currently exist for cultivated tuna, we were not able to assess whether a causal relationship exists between fish mortality and reduced living space. However, we believe that visualization of the trajectories of circling fish using the methods developed in the present study will help increase the efficiency of bluefin tuna cultivation by contributing to future behavioral studies investigating how fish turn at cage walls (Bégout Anras \& Lagardère 2004, Gautrais et al. 2009) and the movement of fish schools in outdoor cages.

The distance at which tagged fish swim from the wall of a net cage remains poorly understood, but we could improve our knowledge of this factor by determining the location of a fish within the cage or assessing the distance of fish trajectories from the wall. To effectively use the method proposed in the present study to do this, several pass points of trajectories should be measured to ensure they are correctly located and mimic the absolute coordinates.

Application of the proposed method may be limited to circling fish in aquaculture, as additional challenges will be met when measuring trajectories of free-ranging aquatic animals in the open sea. However, exact trajectories in the open sea could potentially be estimated if one could remove any drift components that fluctuate periodically, such as tides. Alternatively, if noise sources cannot be identified, acoustic telemetry could be used to detect fish positions within estimated trajectories in the open sea. 
Acknowledgements. We express our sincere gratitude to Dr. Yuichi Tsuda (Fisheries Laboratory, Kinki University), Dr. Katsuya Suzuki (Nittoseimo Co.), Dr. Keigo Ebata, Mr. Eita Ogata, Mr. Shoichi Nagano, Mr. Masataka Marugi (Faculty of Fisheries, Kagoshima University), the staff of Kinki University for providing the specimens required for our study, and 3 anonymous referees for detailed suggestions on improving this manuscript. We sincerely thank Mr. Kanechiku (Kinki University) for his help and support with the NaLA System. We also thank Mr. S. Asaumi (Furuno Electric), Mr. T. Kobayashi (Taiyo A\&F), and the Association of Marino-Forum 21 for their help and support. This work was supported financially by a Grant-in-Aid for Young Scientists (B) (23780200) from the Ministry of Education, Culture, Sports, Science, and Technology and was supported in part by Grants-in-Aid for Scientific Research (C) (22580214) and from the Global COE program 'Centre of Aquaculture Science and Technology for Bluefin Tuna and Other Cultivated Fish' of Kinki University from the Japan Society for the Promotion of Science.

\section{LITERATURE CITED}

Bégout Anras ML, Lagardère JP (2004) Measuring cultured fish swimming behaviour: first results on rainbow trout using acoustic telemetry in tanks. Aquaculture 240:175-186

Bramanti M, Dall'antonia L, Papi F (1988) A new technique to follow the flight paths of birds. J Exp Biol 134:467-472

Gautrais J, Jost C, Soria M, Campo A and others (2009) Analyzing fish movement as a persistent turning walker. J Math Biol 58:429-445

Harcourt RG, Hindell MA, Bell DG, Waas JR (2000) Threedimensional dive profiles of free-ranging Weddell seals. Polar Biol 23:479-487

Hindell MA, Harcourt R, Waas JR, Thompson D (2002) Finescale three-dimensional spatial use by diving, lactating female Weddell seals Leptonychotes weddellii. Mar Ecol Prog Ser 242:275-284

Hiraishi T (2006) Dynamic analysis of cuttlefish basket trap. In: Yamamoto K, Yamane T, Mitsunaga Y (eds) Aquatic biotelemetry and fishing gear telemetry. Kouseisyakouseikaku, Tokyo, p 117-125

Kadota M, White EJ, Torisawa S, Komeyama K, Takagi T (2011) Employing relative entropy techniques for assess-

Editorial responsibility: Tim Dempster, Trondheim, Norway ing modifications in animal behavior. PLoS ONE 6: e28241

Komeyama K, Kadota M, Torisawa S, Suzuki K, Tsuda Y, Takagi T (2011) Measuring the swimming behaviour of a reared Pacific bluefin tuna in a submerged aquaculture net cage. Aquat Living Resour 24:99-105

Kubo T, Sakamoto W, Kumai H (2004) Correlation between oceanic environmental fluctuation and bluefin tuna behavior in the aquaculture pen. Proc Int Symp SEASTAR2000 and Bio-logging Science, December 13-15, 2004, Bangkok, p 92-97

Mitani Y, Sato K, Ito S, Cameron MF, Siniff DB, Naito Y (2003) A method for reconstructing three-dimensional dive profiles of marine mammals using geomagnetic intensity data: results from two lactating Weddell seals. Polar Biol 26:311-317

Okano S, Mitsunaga Y, Sakamoto W, Kumai H (2006) Study on swimming behavior of cultured Pacific bluefin tuna using biotelemetry. Mem Fac Agric Kinki Univ 39:79-82

> Shiomi K, Sato K, Mitamura H, Arai N, Naito Y, Ponganis PJ (2008) Effect of ocean current on the 3-D dive paths of Emperor penguins estimated by dead-reckoning. Aquat Biol 3:265-270

Stockwell RG, Lage WG, Milliff RF (2004) Resonant inertial oscillations in moored buoy ocean surface winds. Tellus Ser A Dyn Meterol Oceanogr 56:536-547

Suzuki K, Torisawa S, Takagi T (2009) Numerical analysis of net cage dynamic behavior due to concurrent waves and current. Proc ASME 2009 28th Int Conf Ocean, Offshore and Arctic Engineering, May 31-June 5, 2009, Honolulu, HI, $\mathrm{p}$ 1-8

> Takagi T, Suzuki K, Hiraishi T (2002) Development of the numerical simulation method of dynamic fishing net shape. Bull Jpn Soc Sci Fish 68:320-326

Wilson RP, Wilson, MP (1988) Dead reckoning - a new technique for determining penguin movements at sea. Meeresforsch Rep Mar Res 32:155-158

> Wilson RP, Grémillet D, Syder J, Kierspel MAM and others (2002) Remote-sensing systems and seabirds: their use, abuse and potential for measuring marine environmental variables. Mar Ecol Prog Ser 228:241-261

Wilson RP, Liebsch N, Davies IM, Quintana F and others (2007) All at sea with animal tracks; methodological and analytical solutions for the resolution of movement. Deep-Sea Res II 54:193-210

Submitted: March 11, 2013; Accepted: May 24, 2013

Proofs received from author(s): June 24, 2013 